\title{
DISEÑO DE HERRAMIENTAS DE ANÁLISIS ARTÍSTICO-MEDIOAMBIENTAL: RESERVA DE LA BIOSFERA DE URDAIBAI COMO CASO DE ESTUDIO
}

\author{
Álvaro Aroca Córdova \\ Universidad del País Vasco / Euskal Herriko Unibertsitatea, Dpto. Arte y Tecnología. \\ Doctorando
}

\section{Resumen}

A partir de la experiencia artística ambiental de la reserva de Urdaibai, la investigación establece indicadores artísticos-medioambientales, técnicos-medioambientales, de impacto ambiental y de sostenibilidad, que permiten determinar y evaluar elementos artísticos- medioambientales de una intervención en las que se da una interacción entre los elementos constituyentes de la obra y un medio natural. Como resultado, se obtuvo un documento de índole sensitivo-artístico-medio ambiental, herramienta de evaluación llamada FEAM "Ficha de Evaluación Artístico Ambiental". En dicha reserva, se desarrollo el proyecto "Sentido y Sostenibilidad", consistente en la intervención de obras "sitio-específicas" de diez artistas. La investigación entregó directrices que sirvieron para mejorar y optimizar la FEAM (Ficha de Evaluación Artístico Medio Ambiental).

Palabras-clave: EVALUACIÓN; SOSTENIBILIDAD; ARTE; MEDIO AMBIENTE; URDAIBAI

\section{ARTISTIC-ENVIRONMENTAL ANALYSIS TOOLS DESIGN: URDAIBAI BIOSPHERE RESERVE AS A CASE STUDY}

\section{Abstract}

Based on the environmental-artistic experience from the Urdaibai's reserve, our research establishes environmental-artistic indicators, environmental-technical indicators and the sustainability and the environmental impact that allow to determinate and evaluate the environmental-artistic elements of the intervention, in which an interaction between the elements of the artwork and the environment takes place. As a result, a document of such sensitive- artistic meaning came out, as a tool of assessment called FEAM "environmental- artistic evaluation form". In this nature reserve, it was developed the project "Sense and Sustainability", which consists of ten site-specific artworks from different artists. The research produced indicators that were useful in order to improve and optimize the FEAM (Environmental-Artistic Evaluation Form).

Keywords: ASSESSMENT; SUSTAINABILITY; ART; ENVIRONMENT; URDAIBAI

\footnotetext{
Aroca Córdova, Álvaro. 2016. “Diseño de herramientas de análisis artístico-medioambiental: Reserva de la Biosfera de Urdaibai como caso de estudio". AusArt 4(1): pp-pp. 77-89 DOI: 10.1387/ausart.16682
}

\section{AUSART}




\section{INTRODUCCIÓN}

Para María Novo $(2002,7)$ "el diálogo entre ciencia y arte es una nueva forma de construir un discurso ambiental integrado e integrador...necesario para la solución de nuevos modelos de desarrollo de nuestro tiempo". Todo ello en el contexto de lograr una transición necesaria a modelos de sociedades más sostenibles ante retos indiscutibles como el cambio climático, o la traslimitación de la huella ecológica planetaria. En este contexto desde hace pocos años se inicia un proceso más crítico, interesado en las realidades ambientales de cada país que incorpora los procesos creativos con evaluaciones ecológicas al mismo nivel. Nos encontramos, por tanto, frente a un ámbito en proceso de desarrollo conceptual, metodológico e instrumental, que debería tender al establecimiento de un proceder acorde con realidades particulares. Esta tendencia se vincula con las propuestas de extensión de las artes y redescubrimiento la naturaleza como motor transformador.

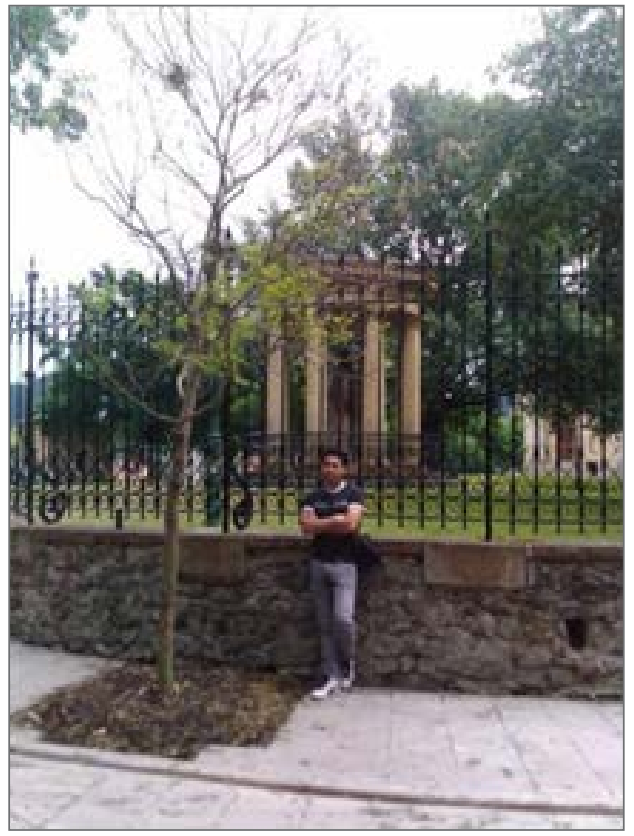

Fig.1: Intervención de la artista Renata Lukas. Gernika. (Julio del 2012).
Antes de relacionar términos artísticos con procesos sostenibles tenemos que tener claro que la idea de desarrollo sostenible se consolidó mediante la publicación del Informe Brutland, también conocido como "Nuestro futuro común" elaborado en 1987 por la Comisión Mundial para el DesarroIlo Sostenible de las Naciones Unidas (Lauces 2004, 4). En este documento la supuesta tensión entre crecimiento económico y protección del medio ambiente se resuelve a través de la conocida idea del desarrollo sostenible, el cual está formado por tres pilares fundamentales imposible de disociar; económico, medioambiental y social.

En este sentido algunos autores establecen que "el desarrollo sostenible o sostenibilidad-sustentable, como lo llaman en Latinoamérica" (Pérez de las Heras 2004, 21) son conceptos iguales 
o similares. Dependerá del contexto el relacionarnos con los términos "sostenibilidad" o "desarrollo sostenible". Ahora bien, "el ecologismo social, en vez de desarrollo sostenible, prefiere hablar de sostenibilidad a secas, sustantivando así el término, ya que este adjetivo se ha venido usando ininterrumpidamente de forma espuria y polémica tras la cumbre de Rio de Janeiro (1992)" (Encina 2014, 30).

$Y$ es a partir de los años sesenta del siglo $X X$ que cambiarán los términos del debate, de manera que la naturaleza pasa de ser objeto de contemplación, a convertirse en sujeto, proceso y destino del hecho artístico. Es decir, se modifica el régimen entre el artista y el medio natural, a través de la experiencia e interacción directa con el mismo; ejercicio en el que también se incluye al espectador. En cualquier caso, debemos considerar que el arte, al igual que la ciencia, la filosofía, la tecnología o la religión, establece un canon mediante el cual, se jerarquiza y clasifica el "orden" del mundo (Caballero 2014, 14).

Para Dark Green Religion (Speranza 2006), "nature is sacred, has intrinsic value, and is therefore due reverent care" ("la naturaleza es sagrada, tiene valor intrínseco, y por eso le merece cuidado reverente", traducción propia), lo que implica, pues, una visión holística o ecocéntrica compartida por muchas tradiciones espirituales de oriente y occidente (Iglesias 2009). En este sentido encontramos algunas analogías de la evolución filosófica de la visión de la naturaleza en algunas culturas no europeas. Por ejemplo, para muchos pueblos indígenas y en especial para el pueblo mapuche, "la noción del cosmos se define por una serie de términos en mapudungun entrelazados con la idea de ser humano, que tienen como eje central el término mapu, que significa la tierra habitada por los che. Ser mapuche es ser del mapu, de la tierra y de esta tierra. Es más que ser originario del lugar, es haber nacido del mapu, y ser parte del mapu a la vez" No cabe en este contexto separar el lugar físico del concepto más amplio de los vínculos de las familias (löf) y de su adscripción territorial (Salas 2009, 45).

\section{ANÁLISIS ARTÍSTICO-MEDIOAMBIENTAL}

La contextualización sensitivo-artística involucra la puesta en valor de una visión tanto personal como social de la intervención artística. De esta forma los objetivos de esta investigación se separaron visualizando un documento 
final que resuma y unifique diferentes puntos de vista de esta investigación artístico-medioambiental los cuales son:

- Establecer indicadores artístico-medioambientales, técnico-medioambientales, de impacto ambiental y de sostenibilidad;

- Determinar y evaluar los elementos artísticos-medioambientales de obras en las que se da una interacción entre los elementos constituyentes de la obra y un medio natural a través de la aplicación de los indicadores;

- Obtener un documento de índole sensitivo-artístico-medioambiental como herramienta de evaluación.

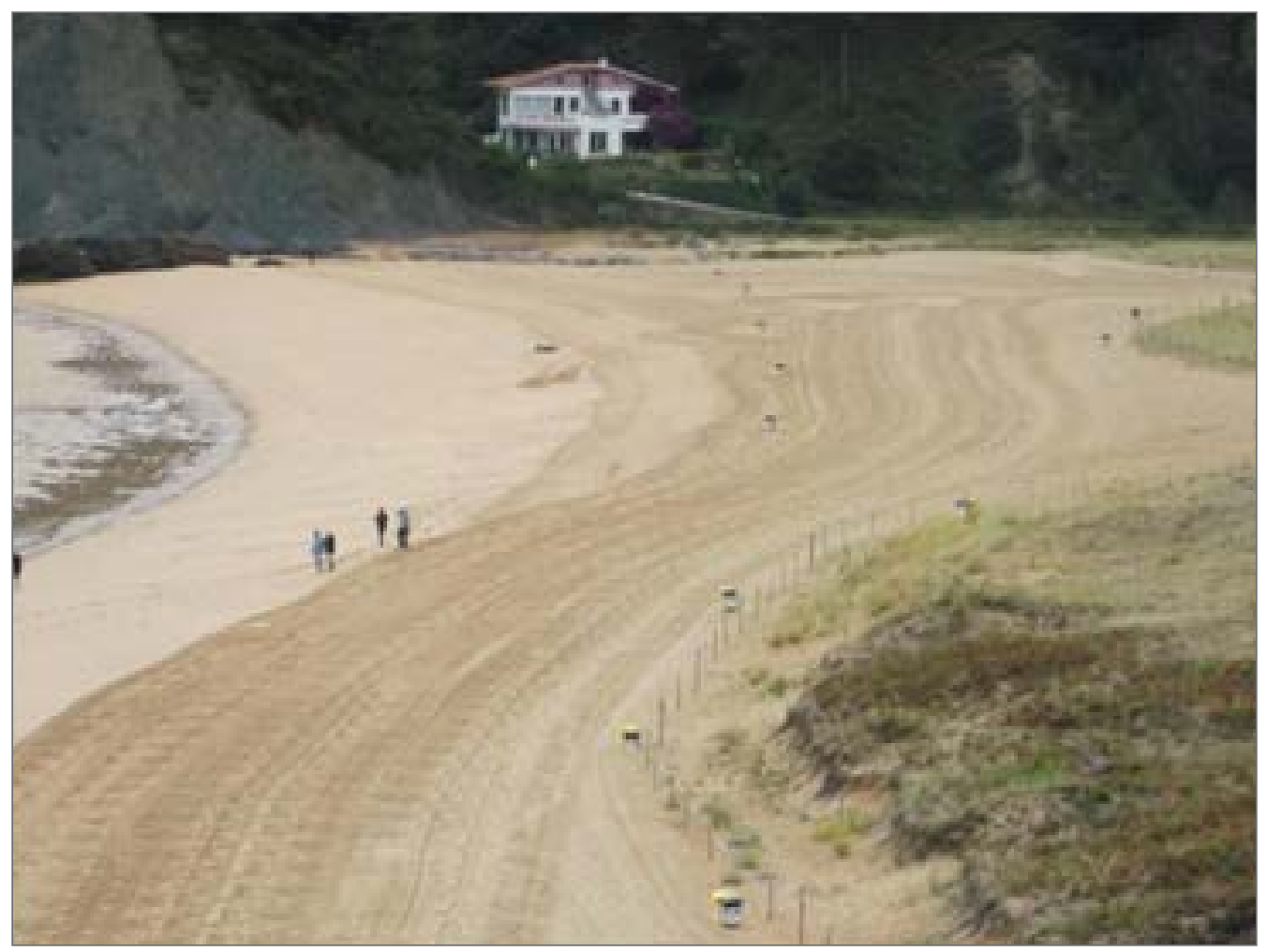

Fig. 2: Intervención de Gunilla Klingberg en la playa de Laga. (Julio del 2012). 
La metodología se basa además en la creación de una ficha de evaluación artístico- medioambiental que compara elementos técnicos de la obra (que tienen relación con su producción, montaje y manutención) y elementos subjetivos (proceso creativo, líneas de trabajo del artista y conocimiento del artista de los lugares de emplazamiento, entre otros). Algunos puntos son:

- Motivaciones personales

- Contexto de Incidencia social

- Paradigma ecológico (Marín 2015)

- Conocimiento de incidencia ambiental de la obra artística

- Influencias de artistas

- Valoración de obra artística en el escenario (in situ) y escenario actual artístico (Arribas 2002, 25)

- Contexto de arte, artista y sociedad

- Proyección de su acción

- Objetivos artísticos de la obra

y datos obtenidos directamente de la información entregada por el artista como:

- Edición del impacto medio ambiental e

- Indicadores de sostenibilidad de obra artística (Quiroga 2001, 47).

Un indicador es un signo, típicamente medible, que puede reflejar una característica cuantitativa o cualitativa, y que es importante para hacer juicios sobre condiciones del sistema actual, pasado o futuro. La formación de un juicio o decisión se facilita comparando las condiciones existentes con un estándar o meta existentes. Tales como: indicadores básicos de sostenibilidad, indicadores de impacto medioambientales, materiales utilizados, contexto del medio natural, análisis de materiales de obra, proceso de creación e instalación en el medio natural, impacto y análisis de la puesta en marcha en medio natural y medioambiental, conservación de la obra de arte en medio natural.

La valoración anterior se entrega según la siguiente figura 3. Además se explican las diferentes siglas que se utilizan y su rango de valores. 


\begin{tabular}{|c|c|}
\hline \multicolumn{2}{|r|}{ Tabla de valoración } \\
\hline 0 & No representa la aseveración \\
\hline 1 & Representa parcialmente la aseveración \\
\hline 2 & Representa la aseveración \\
\hline 3 & Representa muy bien la aseveración \\
\hline UIA & Unidad de Impacto Ambiental varía entre 0 y 1 \\
\hline $\begin{array}{r}\text { Indicadores del } \\
\text { Impacto Ambien- } \\
\text { tal }\end{array}$ & $\begin{array}{l}\text { Un indicador es un signo, típicamente medible, que puede reflejar una } \\
\text { característica cuantitativa o cualitativa, y que es importante para hacer } \\
\text { juicios sobre condiciones del sistema actual, pasado o hacia el futuro. } \\
\text { La formación de un juicio o decisión se facilita comparando las condi- } \\
\text { ciones existentes con un estándar o meta existentes }\end{array}$ \\
\hline UICN & La Unión Internacional para la Conservación de la Naturaleza \\
\hline \multirow{3}{*}{$\begin{array}{r}\text { Categorías de } \\
\text { protección según } \\
\text { las establecidas } \\
\text { por la UICN }\end{array}$} & Categoría I. Protección estricta. Reserva Natural Estricta \\
\hline & $\begin{array}{l}\text { Las áreas protegidas son esenciales para conservar la biodiversidad } \\
\text { natural y cultural y los bienes y servicios ambientales que brindan son } \\
\text { esenciales para la sociedad. A través de actividades económicas, como } \\
\text { el turismo entre otras, muchas áreas protegidas son importantes para el } \\
\text { desarrollo sostenible de comunidades locales, especialmente pueblos } \\
\text { indígenas que dependen de ellos para su supervivencia. }\end{array}$ \\
\hline & $\begin{array}{l}\text { Conservar a escala regional, nacional o global ecosistemas, especies } \\
\text { (presencia o agregaciones) y/o rasgos de geodiversidad extraordina- } \\
\text { rios: dichos atributos se han conformado principalmente o exclusiva- } \\
\text { mente por fuerzas no humanas y se degradarían o destruirían si se } \\
\text { viesen sometidos a cualquier impacto humano significativos. }\end{array}$ \\
\hline
\end{tabular}

Fig. 3: Tabla de Valoración y notas aclaratorias. FEAM. Ficha de Evaluación Artístico-Medio ambiental, Bilbao. (2012).

En una primera etapa, se valora si la intervención requiere o no de una evaluación de impacto ambiental, además de diferentes preguntas referentes a la integración con el contexto medioambiental, materiales de la obra, procesos de creación, montaje, y la conservación de la obra en el medio ambiental. En la figura 3 se muestra la aplicación práctica de la etapa a través del trabajo de campo del artista Gunilla Klingberg (figura 4). 


\begin{tabular}{|c|c|c|}
\hline TÉCNICO-MEDIOAMBIENTAL & $\begin{array}{l}\text { APLICA } \\
\text { SI/NO }\end{array}$ & $\begin{array}{l}\text { VALORACION } \\
\qquad(0-3)\end{array}$ \\
\hline $\begin{array}{l}\text { MEDICION DEL IMPACTO AMBIENTAL DE OBRA ARTISTICA. } \\
\text { ¿Aplica? }\end{array}$ & NO & \\
\hline $\begin{array}{l}\text { CONTEXTO DEL MEDIO AMBIENTAL. Entendiendo que el } \\
\text { contexto medio ambiental no solo se trata donde se desarrolla } \\
\text { la vida sino que también abarca seres vivos, objetos, agua, } \\
\text { suelo, aire y la relación entre ellos. ¿Considera una integración } \\
\text { con del lugar del emplazamiento? }\end{array}$ & SI & 1 \\
\hline $\begin{array}{l}\text { INTEGRACION EN EL MEDIO SOCIAL. Como parte del con- } \\
\text { texto medioambiental }\end{array}$ & SI & 1 \\
\hline $\begin{array}{l}\text { INTEGRACION EN EL MEDIO NATURAL. Como parte del con- } \\
\text { texto medioambiental }\end{array}$ & SI & 1 \\
\hline $\begin{array}{l}\text { MATERIALES DE LA OBRA. ¿Se considera la utilización de } \\
\text { materiales reutilizables o materiales que no contaminen o que } \\
\text { no representen un peligro mínimo el lugar de emplazamiento? }\end{array}$ & SI & 0 \\
\hline $\begin{array}{l}\text { PROCESO DE CREACION. ¿La creación in situ de la obra con- } \\
\text { sidera los procesos de montaje y desmontaje de la obra? }\end{array}$ & SI & 1 \\
\hline $\begin{array}{l}\text { MONTAJE EN EL MEDIO NATURAL. ¿Considera el artista un } \\
\text { impacto mínimo del montaje de la obra en un medio natural? }\end{array}$ & SI & 0 \\
\hline $\begin{array}{l}\text { CONSERVACION DE LA OBRA EN EL MEDIO NATURAL. En } \\
\text { el caso que fuese necesario ¿Se considera la conservación en } \\
\text { el tiempo de la obra en el medio natural? }\end{array}$ & NO & \\
\hline
\end{tabular}

Fig. 4: FEAM. Ficha de Evaluación Artístico-Medio ambiental. La tabla muestra la evaluación Técnico- Medio ambiental. La valoración en las columnas ejemplifica el caso de estudio de la intervención de la artista GuniIla Klimber (Playa de Laga, Urdaibai, 2012).

En esta etapa de la FEAM, se inicia la valoración desde un contexto artístico. Desde las motivaciones personales del artista a través de una entrevista, pasando por la ubicación de la obra en un contexto histórico del arte contemporáneo, en el proyecto sentido y sostenibilidad, la incidencia social y el paradigma ecológico hasta la proyección de la intervención en el contexto correspondiente. 


\begin{tabular}{|c|c|c|}
\hline ARTISTICO - MEDIOAMBIENTAL & $\begin{array}{l}\text { PLICA } \\
\text { SI/NO }\end{array}$ & $\begin{array}{l}\text { VALORA- } \\
\text { CION (0 -3) }\end{array}$ \\
\hline $\begin{array}{l}\text { MOTIVACIONES PERSONALES. ¿Cual fue desde el punto de } \\
\text { vista del proyecto "Sentido y Sostenibilidad" la motivación para } \\
\text { tu obra? (Entrevista a artista) }\end{array}$ & SI & 0 \\
\hline $\begin{array}{l}\text { CONTEXTO HISTORICO EN EL ARTE COMTEMPORANEO } \\
\text { (Medioambiental).Ubicación de la obra en el contexto del arte } \\
\text { contemporáneo, teniendo en cuenta que Urdaibai es Reserva } \\
\text { de la Biosfera }\end{array}$ & SI & 0 \\
\hline $\begin{array}{l}\text { CONTEXTO DE LA OBRA EN EL PROYECTO " SENTIDO Y } \\
\text { SOSTENIBILIDAD. La Obra forma parte de la línea de trabajo } \\
\text { del artista? ¿Es su primera intervención un contexto natural? }\end{array}$ & SI & 0 \\
\hline $\begin{array}{l}\text { CONTEXTO DE INCIDENCIA SOCIAL. ¿Cómo se expresa el } \\
\text { mensaje social de la obra, sabiendo que los mensajes que se } \\
\text { derivan de la obra artística, pueden incidir más directamente en } \\
\text { la población, disponiéndola a favor o en contra de una ade- } \\
\text { cuada gestión de la Naturaleza? }\end{array}$ & SI & 0 \\
\hline $\begin{array}{l}\text { PARADIGMA ECOLOGICO. Replanteamiento de las relacio- } \\
\text { nes cultura/Naturaleza. Concienciación sobre la necesidad de } \\
\text { adoptar pautas nuevas de comportamiento social/ individual. } \\
\text { Cuestionamiento del modelo socioeconómico de la cultura del } \\
\text { consumo. Sensibilidad ecológica ante los problemas medioam- } \\
\text { bientales. }\end{array}$ & SI & 0 \\
\hline $\begin{array}{l}\text { CONOCIMIENTO DEL ARTISTA DE INCIDENCIA AMBIENTAL } \\
\text { DE LA OBRA ARTISTICA. ¿Favorece (o no) tu obra la riqueza } \\
\text { ecológica de la reserva de la Biosfera Urdaibai? }\end{array}$ & SI & $\begin{array}{l}\text { Murieron } \\
\text { arboles y eso } \\
\text { no representa } \\
\text { un mensaje }\end{array}$ \\
\hline $\begin{array}{l}\text { INFLUENCIA DE ARTISTAS EN LA OBRA. ¿Si fuera el caso, } \\
\text { qué tipo de referencias teóricas, artísticas, etc. se manejaron } \\
\text { para llevar a cabo la propuesta? }\end{array}$ & SI & 0 \\
\hline $\begin{array}{l}\text { VALORACION DE OBRA ARTISTICA EN EL ESCENARIO (IN } \\
\text { SITU). Contexto de arte, artista y sociedad. Proyección de su } \\
\text { acción en el tiempo. }\end{array}$ & SI & $\begin{array}{l}\text { La obra no } \\
\text { tiene proyec- } \\
\text { ción }\end{array}$ \\
\hline
\end{tabular}

Fig.5: FEAM. Ficha De Evaluación Artístico-Medioambiental. Metodología de Estudio de Impacto Ambiental Battelle-Columbus. Parte análisis ARTISTICO - MEDIOAMBIENTAL. La valoración en las columnas ejemplifica el caso de estudio de la intervención del artista Renata Lukas (Gernika, 2012).

La medición de Impacto ambiental de una intervención artística (o no) es un proceso que se compone de diferentes indicadores, si bien requiere de una gran cantidad de información. Para nuestro caso de estudio se utilizó una evaluación simplificada. De esta manera se plantea un modelo de trabajo que puede ser mejorado pero que ejemplifica una evaluación en el tiempo con 
el montaje de la obra y sin ella. Así, la última columna indica la diferencia de la valoración. Si el valor es cero no hubo impacto, si la diferencia es positiva hubo impacto y si es negativa nos dice que la intervención es una mejora para el medio.

\begin{tabular}{|c|c|c|c|c|}
\hline \multicolumn{2}{|c|}{$\begin{array}{l}\text { Indicadores del Impacto Ambiental. } \\
\text { METODO DE BATTELLE - COLUMBUS }\end{array}$} & \multirow[t]{2}{*}{$\begin{array}{l}\text { UIA CON } \\
\text { PROYECTO } \\
\text { ARTISTICO }\end{array}$} & \multirow[t]{2}{*}{$\begin{array}{l}\text { UIA SIN } \\
\text { PROYECTO } \\
\text { ARTISTICO }\end{array}$} & \multirow{2}{*}{$\begin{array}{c}\text { DIFEREN } \\
\text { CIA UIA } \\
\text { (CP) - } \\
\text { UIA(SP) }\end{array}$} \\
\hline CATEGORIAS & COMPONENTES & & & \\
\hline \multirow{2}{*}{ Ecología } & Hábitat y comunidades & 0 & 0 & 1 \\
\hline & Ecosistemas & 0 & 0 & 0 \\
\hline \multirow{4}{*}{ Contaminación } & Agua & 0 & 0 & 0 \\
\hline & Atmosfera & 0 & 0 & 0 \\
\hline & Suelo & 0 & 0 & 0 \\
\hline & Ruido & 0 & 0 & 1 \\
\hline \multirow{6}{*}{$\begin{array}{l}\text { Aspectos } \\
\text { Estéticos }\end{array}$} & Suelo & 0 & 0 & 0 \\
\hline & Aire & 0 & 0 & 0 \\
\hline & Agua & 0 & 0 & 0 \\
\hline & Biota & 0 & 0 & 0 \\
\hline & Objetos Artesanales & 0 & 0 & 0 \\
\hline & Composición & 0 & 0 & 0 \\
\hline \multirow{5}{*}{$\begin{array}{l}\text { Aspectos } \\
\text { de interés } \\
\text { humano }\end{array}$} & $\begin{array}{l}\text { Valores Educacionales } \\
\text { y científicos }\end{array}$ & 0 & 0 & 0 \\
\hline & Valores Históricos & 0 & 0 & 0 \\
\hline & Valores Culturales & 0 & 0 & 0 \\
\hline & Sensaciones & 0 & 0 & 0 \\
\hline & Estilos de Vida & 0 & 0 & 0 \\
\hline
\end{tabular}

Fig.6: Metodología de Estudio de Impacto Ambiental Battelle-Columbus. FEAM. Ficha de Evaluación Artístico-Medioambiental. La valoración en las columnas ejemplifica el caso de estudio de la intervención de la artista Renata Lukas (Gernika, 2012). 
Los indicadores de sostenibilidad (Figura 7) unen aspectos de la economía local, aspectos sociales e indicadores medioambientales. Al igual que el caso de la evaluación de Impacto medioambiental se simplifico para nuestro caso de estudio. En una investigación profunda estos indicadores entregan en buena medida evaluaciones a mediano y largo plazo, visualizando verdaderamente la incidencia de la intervención artística en el medio. Siendo una herramienta muy útil para una estudio que abarque diferentes campos del conocimiento.

\begin{tabular}{|c|c|c|}
\hline \multicolumn{3}{|c|}{ INDICADORES DE SOSTENIBILIDAD } \\
\hline & Indicador & Medidas especificas \\
\hline 1 & Protección del sitio & $\begin{array}{l}\text { Categorías de protección según las establecidas por la } \\
\qquad \text { UICN }\end{array}$ \\
\hline 2 & Estrés del Lugar & $\begin{array}{l}\text { Número de visitantes a las obras artísticas en la zona (por } \\
\qquad \text { año, por mes) }\end{array}$ \\
\hline 3 & Intensidad de Uso & $\begin{array}{l}\text { Intensidad de uso de la zona afectada por la instalación de } \\
\qquad \text { la obra }\end{array}$ \\
\hline 4 & Impacto Social & Relación visitantes a la obra / residentes \\
\hline 5 & Control del desarrollo & Existencia de procedimientos para un control del desarrollo \\
\hline 6 & Gestión de Residuos & Porcentaje de residuos tratados \\
\hline 7 & $\begin{array}{l}\text { Grado de Planifica- } \\
\text { ción }\end{array}$ & $\begin{array}{l}\text { Existencia de planes organizados para el desarrollo regio- } \\
\qquad \text { nal }\end{array}$ \\
\hline 8 & Ecosistemas Críticos & Número de especies amenazadas \\
\hline 9 & $\begin{array}{l}\text { Satisfacción del } \\
\text { visitante }\end{array}$ & Nivel de satisfacción del visitante de la obra \\
\hline 10 & Satisfacción local & Nivel de satisfacción población \\
\hline 11 & $\begin{array}{l}\text { Contribución a la } \\
\text { economía local }\end{array}$ & $\begin{array}{l}\text { Proporción de la actividad que produce la visita a las obras } \\
\qquad \text { artísticas en la economía local }\end{array}$ \\
\hline
\end{tabular}

Fig.7: FEAM. Ficha de Evaluación Artístico-Medio ambiental. Indicadores de Sostenibilidad (Bilbao 2012). 


\section{CONCLUSIONES}

La investigación logró establecer indicadores artísticos-medioambientales, técnicos- medioambientales, de impacto ambiental y de sostenibilidad, determinando y evaluando los elementos artísticos-medioambientales de la intervención en las que se da una interacción entre los elementos constituyentes de la obra y un medio natural, a través de la aplicación de los indicadores.

Se consiguió obtener un documento de índole sensitivo-artístico-medio ambiental como herramienta de evaluación que se llamo FEAM "Ficha de Evaluación Artístico Ambiental". Un número representa la suma entre la evaluación técnico-ambiental y artístico-medioambiental. Una valoración mayor supone una intervención acorde con parámetros medioambientales tanto en un contexto social, natural, materiales de la obra, procesos de creación y conservación en el medio natural. A la valoración anterior es importante agregar que una parte de la FEAM incluye una entrevista al artista, mediante correo electrónico, video llamada u otra herramienta. Las evaluaciones de Impacto Ambiental para las intervenciones artísticas se realizaron desde un punto de vista cualitativo. Esto supone que en la medida que un proyecto de intervención en el medio natural se geste, se debe realizar un seguimiento a nivel de todos los procesos de creación, montaje y conservación en el medio donde se emplaza. Para realizar un verdadero uso de las herramientas que nos entrega la Ficha de evaluación artístico ambiental, es necesario realizar estudios de campos en donde todos los protagonistas sean consultados y expresen su voluntad de participar en la medida de lo posible. Desde comisarios, organizadores y artistas. Son las propias acciones previas desde la gestación conceptual de la obra hasta su montaje y conservación, que nos entregarán un ordenamiento y valoración real de los indicadores velando por un trabajo que pueda sostenerse por sí mismo y entregue una vinculación e interacción con lo que le rodea.

En efecto, todas las intervenciones artísticas tenían un marco conceptual muy amplio con fundamentos profundos. Algunas muy concretas y otras demasiado "sofisticadas" cayendo en el "olvido de Urdaibai". Para Dieter Roelstraete (2012), Pieter Vermeersch es "...un artista demasiado inteligente para creer que el arte puede responder correctamente a lo sublime negativo de las desgracias del mundo (y mucho menos "hacer" algo con ello), pero también demasiado sofisticado para rechazar de manera inexpresiva nuestra obligación común, como mínimo, de reflexionar sobre estas cuestiones". 
Con los distintos puntos de vista que trata de abarcar la FEAM, es posible tener una visión panorámica de este tipo de intervenciones que se desarrollan en un medio natural. Además, es inevitable contemplar que existen muchos vínculos entre arte, medioambiente, sociedad, cultura y economía, los cuales están en una profunda interacción constantemente. De esta forma las conclusiones responden a los diferentes objetivos abriendo nuevas visiones en este complejo mundo de arte y ciencia.

\section{Referencias bibliográficas}

Albelda Raga, José Luís \& José Rafael Saborit Viguer. 1997. La construcción de la Naturaleza. València: Direcció General de Promoció Cultural, Museus i Belles Arts

Arrhenuis, Sara. 2012. "Los estratos del tiempo: Gunilla Klingberg, A sign in space". En: Zentzua eta iraunkortasuna : Irakurgaiak [Sentido y sostenibilidad : Lecturas...], 123-8. Vitoria-Gasteiz: Fundación 2012

Arribas Navarro, Diego, coord. 2002. Arte, industria y territorio: Minas de Ojos Negros (Teruel). Teruel: Artejiloca

Dussel Ambrosini Enrique, Eduardo Mendieta \& Carmen Bohórquez-Morán, eds. 2009. El pensamiento filosófico latinoamericano, del Caribe y latino (1300-2000): Historia, corrientes, temas y filósofos. México DF: Siglo XXI

Encina Rodríguez, Javier \& Iñaki Barcena Hinojal. 2004. "Democracia ecológica, formas y experiencias de participación en la crisis ambiental". Sevilla: UNILCO

Iglesias, Elisa, 2009. "La obra de Arne Naess, rica en elementos para la transformación cultural". En "Ecología profunda", número monográfico El Ecologista 61. https://www.ecologistasenaccion.org/article20342.html

Lauces Méndez, Pilar. 2004. "Sensibilización medioambiental: Conceptos básicos y problemas globales". Madrid: Ideas Propias

Marín Ruiz, Carmen. 2015. "Arte medioambiental y ecología: Paradigmas de comprensión, interpretación y valoración de las relaciones entre arte y ecología (1960-2015) [Enviromental Art and Ecology: Paradigms for understanding, interpreting and evaluating the relationship between Art and Ecology (1960-2015)]. Tesis Univ. del País Vasco

Novo Villaverde, María, coord. 2002. "Ciencia, Arte y medioambiente". Madrid: Mundi Prensa

OSE (Observatorio de la Sostenibilidad en España). 2012. Sostenibilidad en España 2012: Energía sostenible para todos. Madrid: Ministerio de Agricultura, Alimentación y Medio Ambiente

Oyarzún Robles, Pablo. 1979. Anestética del ready-made. Libros de la Invención y de la Herencia. Santiago de Chile: LOM

Pérez de las Heras, Mónica. 2004. Manual del turismo sostenible: Cómo conseguir un turismo social, económico y ambientalmente responsable. Madrid: Mundi-Prensa

Punín Burneo, María Gabriela, María José Valarezo \& Ricardo Pavel Ferrer. 2014. "Arte, microorganismos y Ciencia". Arte y Políticas de Identidad 10-11 
Quiroga Martínez, Rayen. 2001. "Indicadores de sostenibilidad ambiental y de desarrollo sostenible: Estado del arte y perspectivas". Santiago de Chile: CEPAL

Roelstraete, Dieter. Edmund Burke e Immanuel Kant, paseando juntos en Urdaibai : Pieter Vermeerssch, Untitled". En: Zentzua eta iraunkortasuna : Irakurgaiak [Sentido y sostenibilidad : Lecturas...], 155-60. Vitoria-Gasteiz: Fundación 2012

Salas Astrain, Ricardo. 2009. "Hermeneutic philosophy and human experience: An intercultural perspective of worldviews and life world in the Mapuche culture". En Worldviews and Cultures: Philosophical Reflections from an Intercultural Perspective, eds: N. Note et al., 16379. Einstein Meets Magritte: Interdisciplinary Reflection on Science, Nature, Art, Human Action and Society 10. Berlin: Springer

Sánchez Balmisa, Alberto et al. 2012. Zentzua eta iraunkortasuna : Irakurgaiak [Sentido y sostenibilidad : Lecturas; Bon sens et durabilité : Lire; Sense and sustainability : Reading]. Intervenciones específicas en la naturaleza en la Reserva de la Biosfera de Urdaibai (21 julio-23 de septiembre) en el marco de "2012 Euskadi, Año de las Culturas por la Paz y la Libertad". Vitoria-Gasteiz: Fundación 2012

Speranza Andrea. 2006. Ecología profunda y autorrealización: Introducción a la Filosofía ecológica de Arne Naess. Buenos Aires: Biblios

(Artículo recibido 30.04.16; aceptado 19.05.16) 